\title{
A murine model of interlamellar corneal transplantation
}

\author{
Chun Ho Lau, Susan M Nicholls, David L Easty
}

\begin{abstract}
Aims/background-There are more reagents and information available for immunological studies in the mouse compared with other animals. Unfortunately, the mouse penetrating keratoplasty model is associated with high background inflammation which hinders study of the immune response to the graft. To mitigate this drawback, a murine orthotopic corneal interlamellar transplantation model with mild non-specific inflammation was developed.
\end{abstract}

Methods-A $1.5 \mathrm{~mm}$ diameter full thickness donor corneal button was placed in a $2 \mathrm{~mm}$ diameter recipient corneal interlamellar pocket without placement of a suture. The clinical course of graft status was studied daily for 60 days in 30 allografts (donor strain CBA $101\left(\mathrm{H}-2^{\mathrm{k}}\right)$ to recipient NIH $\left(\mathrm{H}-2^{q}\right)$ ) and 30 syngeneic grafts (NIH to NIH) by slit lamp biomicroscopy and scored for neovascularisation, opacity, oedema, and granularity. In another cohort of animals, histological observation was performed after $\mathbf{3 0}$ minutes and on days $10,20,30$, and 40 after transplantation (four allografts and four syngeneic grafts per time point). Histological study was also performed on grafts without donor epithelium and on interlamellar pockets without grafts.

Division of

Ophthalmology,

Medical School,

University of Bristol,

University Walk,

Bristol BS8 1TD

C H Lau

S M Nicholls

Division of

Ophthalmology,

Bristol Eye Hospital,

Lower Maudlin Street,

Bristol BS1 2LX

C H Lau

D L Easty

Correspondence to: Dr Chun Ho Lau, Division of Ophthalmology, Bristol Eye Hospital, Lower Maudlin Street, Bristol BS1 2LX.

Accepted for publication 10 October 1997 tion.

(Br F Ophthalmol 1998;82:294-299)
Immunological rejection is the commonest cause of corneal graft failure. ${ }^{12}$ Since Maumenee showed in 1951 that corneal graft rejection was an acquired immunity, ${ }^{3}$ many animal models of human rejection have been described and have been reviewed by Niederkorn. ${ }^{4}$ They vary from heterotopic corneal transplantation to orthotopic interlamellar keratoplasty, orthotopic lamellar keratoplasty, and orthotopic penetrating keratoplasty. The rabbit model $^{35}$ was initially popular but lost favour because of paucity of immunological information and reagents for this species, as well as the lack of inbred strains. With the advancement of sutures and operating microscope the rabbit was gradually superseded by the rat $^{67}$ and, more recently, the mouse. ${ }^{8-10}$ For many reasons, including the availability of many inbred congeneic mouse strains with defined MHC genes and the presence of abundant immunological information and reagents, the mouse is one of the most useful models for immunological studies.

For transplantation, however, the mouse penetrating keratoplasty (PK) model $^{10}$ has some important drawbacks. The anterior chamber is very shallow and collapses once opened. The procedure is prone to high surgical failure rate and surgical complications such as hyphaema, anterior synechiae, and iatrogenic cataract are common. Because of the relatively small body mass of the mouse (about $25 \mathrm{~g}$ ) compared with that of rat (about $150 \mathrm{~g}$ ) and rabbit (about $2.5 \mathrm{~kg}$ ), relatively greater surgical trauma is induced. In particular, the presence of at least eight full thickness sutures of similar size to those used in humans and the requirement of similar operation time are associated with extremely high background inflammatory responses. A histological pilot study using the mouse PK model revealed that there was persistent heavy cellular infiltration especially around sutures, in both syngeneic grafts and allografts (data not shown). This infiltration masks clinical observation so that rejection cannot be reliably defined. To avoid this major problem, a number of approaches have been adopted by other investigators. Scores of different clinical variables have been summed in order to reveal significant difference in response between allografts and syngeneic grafts. ${ }^{10}$ Sutures have been removed at an early phase. ${ }^{11}{ }^{12}$ However, in our experience, by the 

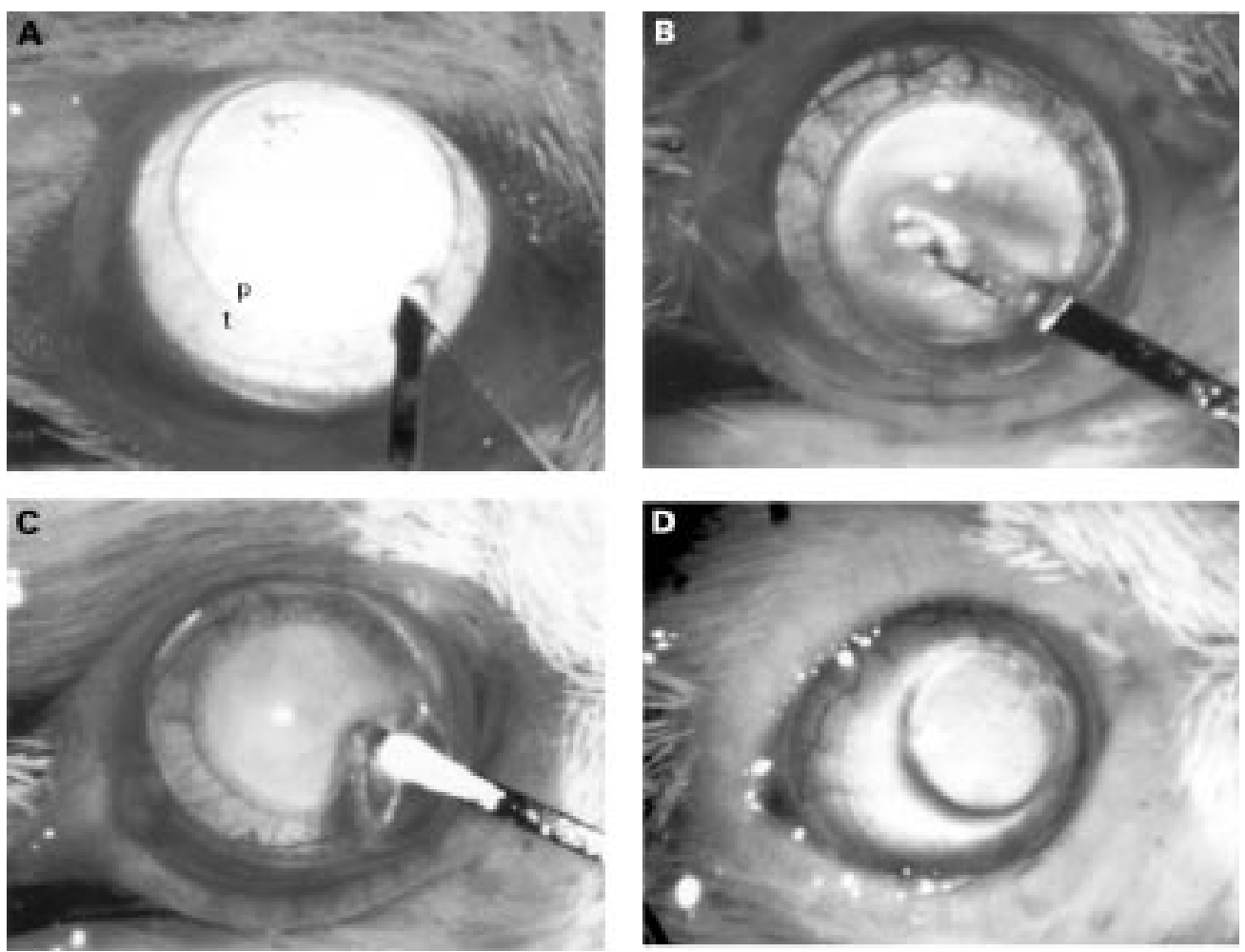

Figure 1 Transplantation procedure. (A) Cornea was marked with a $2 \mathrm{~mm}$ trephine, a small incision was made inferonasally, $p=$ pupil margin, $t=$ trephine mark. (B) An interlamellar tunnel was fashioned with a microblade, followed by interlamellar dissection. (C) After enlarging the incision with scissors from 3 to 6 o'clock, a 1.5 mm full thickness donor button was introduced into the pocket. (D) The graft remained in the pocket without use of a suture.

time of suture removal, the background inflammation is already quite severe and the process of suture removal itself induces inflammation. In general, grafts have been assessed only on a weekly basis. ${ }^{10-12}$ In such circumstances, crucial events might be missed.

To avoid these limitations, a murine orthotopic corneal interlamellar transplantation model with mild non-specific inflammation was developed. To assess the effect of donor epithelium on the formation of interlamellar epithelial cyst and rejection, grafts with or without donor epithelium were transplanted.

\section{Materials and methods}

MICE

Inbred female CBA $101\left(\mathrm{H}-2^{\mathrm{k}}\right)$ mice were bred in the animal facilities at Bristol University. $\mathrm{NIH}\left(\mathrm{H}-2^{\mathrm{q}}\right)$ female mice were obtained from Harlan UK Limited (Bicester, UK). Mice were aged 8 to 12 weeks at the time of transplantation.

METHOD OF GRAFTING

Recipients were anaesthetised by subcutaneous injection of $0.15 \mathrm{mg} / \mathrm{kg}$ fentanyl citrate and 4.5 $\mathrm{mg} / \mathrm{kg}$ fluanisone (Hypnorm) (Janssen Pharmaceutical, Grove, UK) and $2 \mathrm{mg} / \mathrm{kg}$ midazolam hydrochloride (Hypnovel) (Roche Products, Welwyn Garden City). After topical anaesthesia with $1 \%$ amethocaine hydrochloride (tetracaine) (Chauvin Pharmaceuticals Ltd, Romford), the operation was carried out under the hydration of balanced salt solution (BSS). Figure 1 shows the steps of grafting. The eye was held between the eyelids with inverted Collibri forceps. A shallow $2 \mathrm{~mm}$ diameter mark coaxial with the pupil was impressed on the cornea with a trephine. A small incision was made inferonasally to create an entry from epithelium into mid stroma. An interlamellar tunnel was fashioned with a microblade adapted from the needle of a $10-0$ suture and fixed at the end of a wooden handle. Interlamellar dissection was completed by gradual lateral sweeping motion of the microblade until the $2 \mathrm{~mm}$ diameter mark was reached in all directions. The incision was then enlarged with scissors from 3 to 6 o'clock to form a $90^{\circ}$ entry at the inferonasal quadrant. Next, a $1.5 \mathrm{~mm}$ full thickness donor cornea button was trephined from the donor under the hydration of BSS. The donor button was then introduced into the interlamellar pocket with donor epithelium facing upwards. The graft remained in the pocket without use of a suture. The eye was then covered with chloramphenicol ointment.

\section{REMOVAL OF DONOR EPITHELIUM FROM THE} GRAFT

To remove the donor epithelium completely, the following procedure was adopted. Shallow anterior interlamellar dissection was performed on anaesthetised donors as described above. Donors were then killed by cervical dislocation. A $1.5 \mathrm{~mm}$ full thickness donor cornea button was trephined from each eye under the hydration of BSS. The anterior lamella with attached epithelium was discarded. The thicker posterior lamella (completely free of 
Table 1 Scoring system for graft status

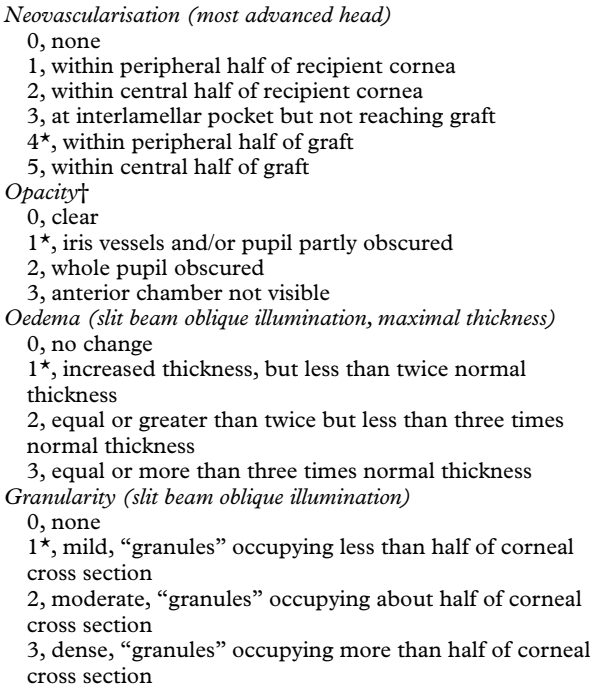

${ }^{\star}$ Minimal score for each variable by which clinical rejection was defined.

†Adapted ${ }^{11}$ with modification.

donor epithelium) was then used as the epithelium-free graft.

\section{FOLLOW UP OF GRAFT STATUS}

After transplantation, graft status was followed daily by slit lamp biomicroscopy for clinical signs of rejection until the end of the clinical study or up to the time of histological observation. The central graft with surrounding recipient bed was scored for vascularity, opacity, oedema, and granularity (granularity meaning the grain-like appearance of the stroma, which was interpreted as small foci of infiltrating cells) (Table 1). Grafts were defined as rejected when score combinations were at least 4 for vascularisation, 1 for opacity, 1 for oedema, and 1 for granularity. After observation, the eye was covered with chloramphenicol ointment for the first 7 days.

HISTOLOGY

Eye specimens were fixed overnight in $10 \%$ formal saline, then automatically dehydrated and infiltrated with wax (Reichert-Jung Histokinette 2000, Leica, Germany). Specimens were embedded in paraffin and stored at $-20^{\circ} \mathrm{C}$. When required, $6 \mu \mathrm{m}$ sections were cut onto glass slides which were stained by a standard haematoxylin and eosin procedure.

EXPERIMENTAL PROTOCOL

For study of the course of clinical rejection, CBA 101 strain corneas or NIH strain corneas were transplanted to NIH strain recipients. Sixty transplants were performed (30 allografts and 30 syngeneic grafts). Animals were coded so that the graft status could be followed up in a masked fashion for 60 days. A further cohort of interlamellar transplants was performed for histological observation at 30 minutes and on days 10,20,30, 40 after operation (four pairs of allografts and syngeneic grafts per time point). Histological study was also performed on grafts without donor epithelium (four allografts and four syngeneic grafts per time point) and on interlamellar pockets without grafts on days 10,20,30 (four animals per time point).

\section{STATISTICS}

The Mann-Whitney test was used to compute the $p$ values in the study of clinical course of graft status. A p value of less than 0.05 was deemed significant.

\section{Results}

CLINICAL COURSE OF GRAFT STATUS

The overall complication rate was less than $5 \%$. Interoperative complications included accidental penetration of the anterior chamber or anterior lamella of the pocket and hyphaema. Postoperative complications included secondary hyphaema, cataract, and infection. Such animals were discarded from the study and killed. From pilot studies (data not shown), it was observed that the incision closed rapidly and the accompanying epithelial defect usually disappeared by day 2 or 3 . The donor epithelium grew rapidly inside the pocket, forming an epithelial cyst, which usually became apparent from day 4 or 5 onwards as bead-like opacities at the level of the pocket. The clinical picture of graft rejection (vascularisation, development of opacity, oedema, and granularity) correlated closely with the proximity of vessels to the graft. The granularity observed on slit beam oblique illumination was found to relate to the degree of cellular infiltration as indicated by histological examination of the graft and recipient bed.
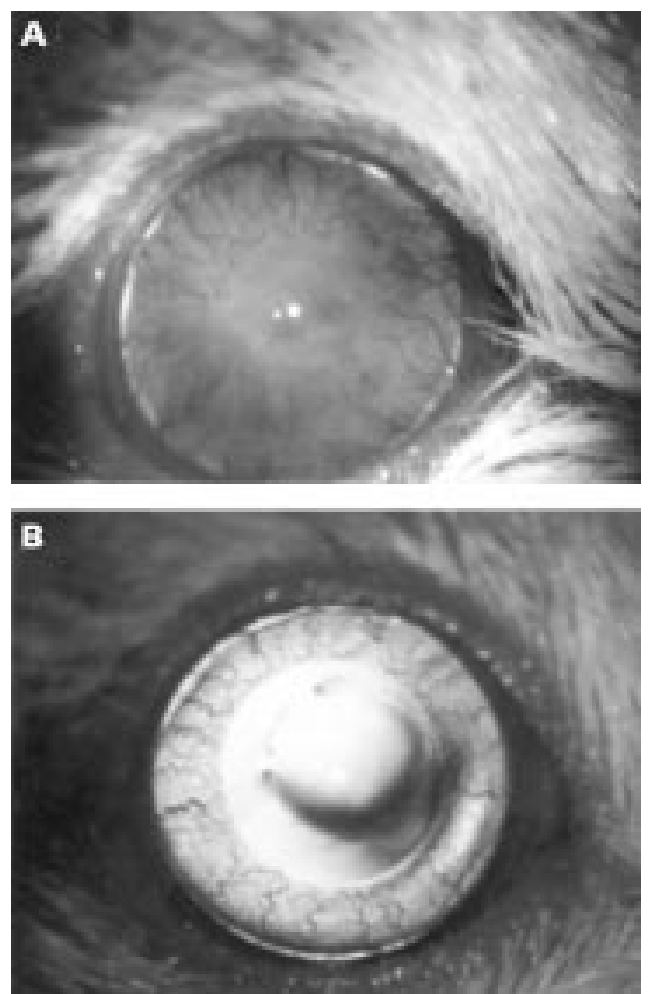

Figure 2 (A) An allograft undergoing rejection on day 25 after transplantation (neovascularisation, 4; opacity, 2; oedema, 1; granularity, 1). There is diffuse milky opacity of the graft obscuring the pupil, and heavy corneal neovascularisation. (B) A clear syngeneic graft, on day 25 after transplantation. There was no neovascularisation. 

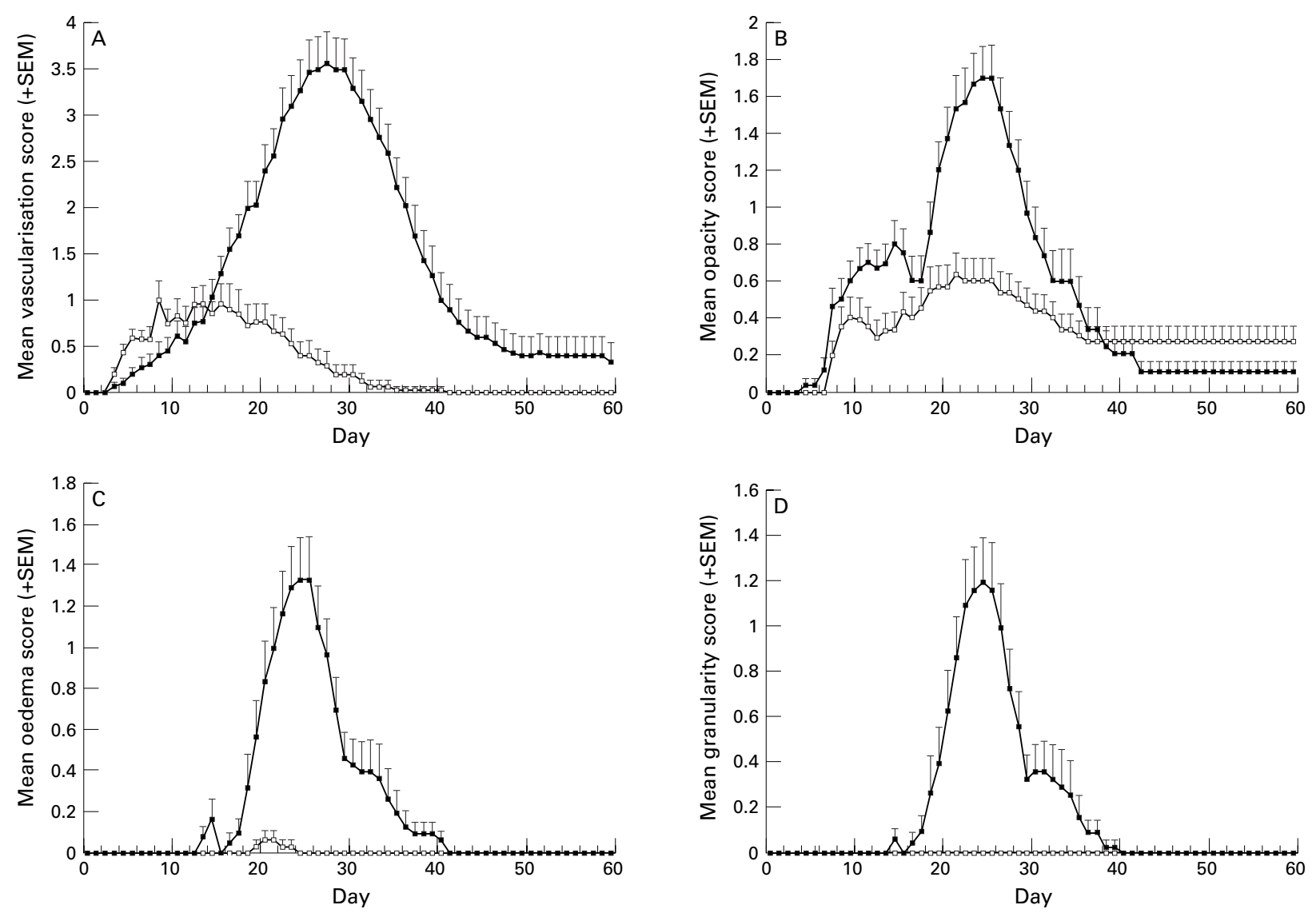

Figure 3 Clinical course of graft status. (A) Neovascularisation score ( $+S E M)(p<0.05$ on days 4-6 and 16-41). (B) Opacity score ( $+S E M)(p$ $<0.05$ on days 11-12, 14, and 19-29). (C) Oedema score ( $+S E M)(p<0.05$ on days 19-32). (D) Granularity score $(+S E M)$ ( $p<0.05$ on days $20-31)$. Solid squares represent allografts, and open squares represent syngeneic grafts.

Figure 2 shows the picture of clinical rejection, and Figure 3 shows the courses of the mean clinical scores after transplantation in the group of 30 allografts and 30 syngeneic grafts. Eighty per cent (24/30) of allografts were defined as rejected by 32 days (median 21 days, range 18 to $>60$ days). The mean neovascularisation score was significantly greater in the syngeneic grafts $(\mathrm{p}<0.01)$ on days $4-6$. However, it became significantly greater in the allografts on days 16-41 ( $\mathrm{p}<0.05)$. After day 28 , neovascularisation began to diminish but never reduced to the level of syngeneic grafts during the period of study. The mean opacity score was significantly greater in the allografts than in syngeneic grafts on days 11-12, 14, 19-29 ( $\mathrm{p}<0.05$ ). After day 38, the opacity score of allografts subsided to a level lower than that of syngeneic grafts. The mean oedema score was significantly greater in the allografts than in syngeneic grafts on days $19-32(\mathrm{p}<0.05)$. Oedema of the allografts had diminished to the level of syngeneic grafts by day 41. The mean granularity score of allografts was significantly greater than that of syngeneic grafts on days $20-31(\mathrm{p}<0.05)$, but had diminished to that of syngeneic grafts by day 40 .

A similar course of clinical rejection was observed in the cohort used for histological observation to that in the clinical course study. Clinical rejection was not observed if the graft epithelium was removed or after interlamellar dissection without grafts.

HISTOLOGICAL OBSERVATION

By 30 minutes after transplantation, the endothelium of the graft was still intact and separation between graft and recipient bed was obvious (Fig 4A). By day 10, donor endothelial cells had disappeared from both allografts and syngeneic grafts. Graft epithelium was present in the pocket at all time points in syngeneic grafts and in allografts that had not been defined as clinically rejected. In all grafts, the epithelium of the graft spread rapidly to line the "ceiling" of the pocket, forming epithelial cysts. Heavy mixed cell infiltration consisting of round cells (probably lymphocytes), cells with a more dendritic morphology, and neutrophils (that is, cells with an irregular nucleus), was observed in the graft and recipient bed only in allografts. This was accompanied by destruction and disappearance of the graft epithelial structure (Fig $4 \mathrm{~B})$. In syngeneic grafts, usually no infiltration was detected in the graft and the epithelial cyst remained intact (Fig 4C), although mild cell infiltration might be seen in the peripheral cornea of the recipient up to the graft recipient junction.

After interlamellar dissection alone, there was no epithelium in the pocket at any time point. The stromal interlamellar pocket was obvious at 30 minutes after operation, but 


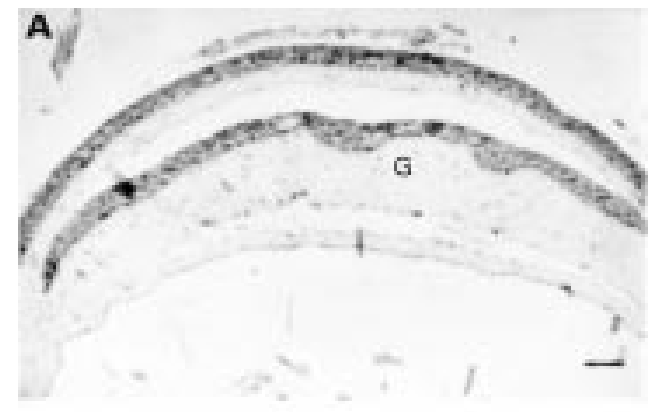

B
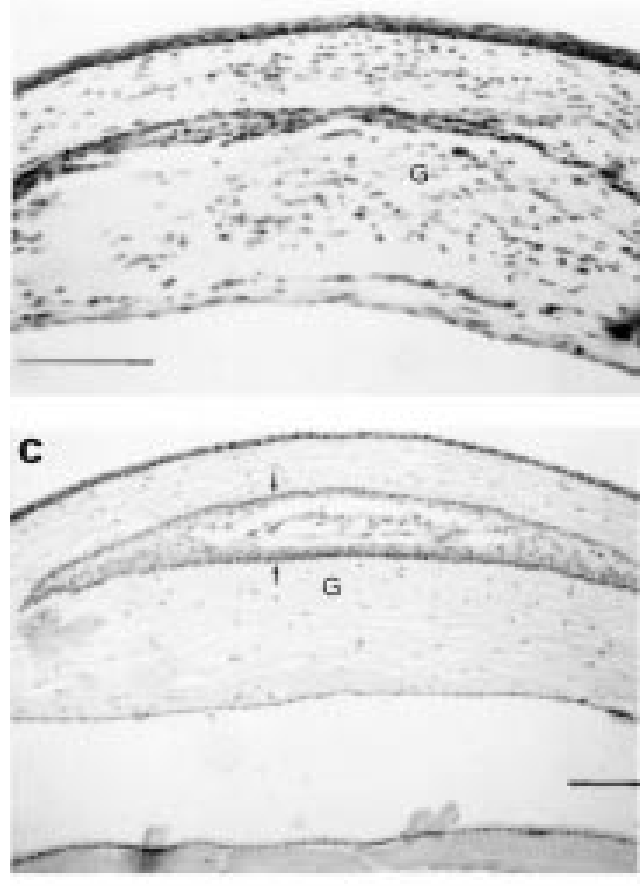

Figure 4 Histological appearance of grafted corneas. $G=$ graft. (A) 30 minutes after transplantation; there is obvious separation between recipient and graft above and below. (B) 30 days after transplantation; an allograft showing dense cell infiltration with disappearance of some of the donor epithelium. (C) 30 days after transplantation; an intact syngeneic graft except for disappearance of donor endothelium. There is a fully developed epithelial cyst (arrows) but no cell infiltration. (Haematoxylin and eosin, bar represents $200 \mu \mathrm{m}$.)

became unobservable by day 10 . When the graft button was transplanted without epithelium, there was no epithelium observed in the pocket at any time point. At 30 minutes after grafting, the separation between recipient bed and allograft was still obvious. Donor stromal structure and keratocytes were present at all time points. There was no significant cell infiltration in grafts lacking epithelium.

\section{Discussion}

We have shown that corneal interlamellar keratoplasty is feasible in the mouse. In the strains combination that we used, rejection could be clearly defined and occurred in $80 \%$ of allografts by day 32 . The $80 \%$ incidence of rejection in the clinical study is comparable with that reported by some ${ }^{10}$ but higher than in others $^{11}{ }^{12}$ in the murine PK model. Perhaps this was partly due to the fact that the strains combinations as well as MHC disparities were different in these studies. In the rat model, similar graft failure rates were observed in both penetrating and interlamellar keratoplasty in the same strain combination. ${ }^{13}$ Because in the series of interlamellar keratoplasty without graft epithelium, there was no case of clinical rejection, it was concluded that the allogeneic donor epithelium was the major target of the rejection process.

A significant increase in neovascularity was usually followed sequentially by a significant increase in opacity, oedema, and granularity, indicating that all these variables were correlated with rejection. Both oedema and granularity were mild in the syngeneic grafts. The pocket microenvironment supported the persistence and proliferation of the donor epithelium, which was shown histologically by the epithelialisation of the ceiling of the pocket, forming a cyst, as reported in the rat interlamellar model. ${ }^{14}$ Although it was not proved that the epithelial cyst was entirely of donor origin, the lack of appearance of epithelium or epithelial cysts in pockets without graft or in grafts without epithelium suggested that the cyst was not formed by recipient epithelial ingrowth. The opacity caused by the epithelial cyst could be differentiated from that of rejection by the fact that the former was pearl-like and confined to the pocket level, while the latter was diffusely cloudy and the recipient bed was also involved. The opacity in syngeneic grafts was mainly due to this cyst, although a mild cell infiltrate was sometimes seen. A greater share of opacity in the allograft was caused by the cell infiltration, indicating that this infiltration was a rejection response rather than a reaction to the cyst. Continuing opacity after day 32 was due to persistence of the cyst which became relatively more pronounced in the syngeneic grafts because the donor epithelium was not destroyed by rejection. The persistent vascularisation in the allografts was presumably due to the time lag required for vessels to regress after rejection was complete.

Consistent with the clinical findings, the histological observation revealed moderate to heavy cell infiltration only in allografts, with destruction of the epithelial cyst and sparing of recipient epithelium and endothelium, confirming that genuine immunological rejection was taking place. The histology of allografts without heavy cellular infiltration and that of syngeneic grafts were very similar.

It is clear that the main target of rejection in this model is the epithelium, although in some histological sections few cells remained in the donor stroma, suggesting that donor keratocytes were also rejected. The role played by the epithelium in human rejection has been disputed because of conflicting evidence concerning the value of removing donor epithelium before transplantation. ${ }^{15}{ }^{16}$ However, rejection of the epithelium has been shown in many human and animal studies. ${ }^{17-23}$

Although some donor endothelial cells were present 30 minutes after grafting, their disappearance on day 10 and at later time points 
confirmed that they did not survive in the pocket microenvironment and were clearly not a target of the immune response. However, they were present at the early stage after transplantation and may well have played a role in the sensitisation process.

Although the endothelium is not a target for rejection, this interlamellar model has useful advantages compared with the PK model in mice. The interlamellar keratoplasty was less time consuming and less tedious to perform, the procedure averaging about $15-20$ minutes per keratoplasty, thus increasing the practicability of experimental study on a large number of animals. In the present study, routinely 10 or more transplants could be performed daily. Moreover, traction sutures were not necessary to proptose the eye during the operation and the graft was held in place by the pocket rendering sutures unnecessary. Apart from the incision, the smooth corneal surface was preserved. These characteristics, together with the fact that the anterior chamber was not entered, explain the mild background inflammation associated with the model. It provided a window that was clear enough to allow daily assessment of the graft and define clear rejection end points, a process which is extremely difficult, if not impossible, with a mouse PK model. Such clear definition of rejection is important for study of the role played by effector cells and molecules, especially cytokines, because these may be involved in post-transplantation inflammation as well as the rejection response. Furthermore, the dynamic interactions between effector cells and molecules are usually rapid and cytokine expression is known to be transient. ${ }^{24}$ Therefore, a mouse model in which cytokine production can be closely related to the stage of rejection, both clinically and histologically, is essential for such investigations.

This study was supported by the United Bristol Healthcare NHS Trust. C H Lau was supported by an ORS scholarship from the Overseas Administration Department of HM Government and a bursary from the Hammond Trust.

1 Vail A, Gore SM, Bradley BA, et al. Clinical and surgical factors influencing corneal graft survival, visual acuity, and astigmatism. Ophthalmology 1996;103:41-9.
2 Pleyer U, Steuhl KP, Weidle EG, Lisch W, Thiel H-J. Corneal graft rejection: Incidence, manifestation, and interaction of clinical subtypes. Transplant Proc 1992;24: 2034-37.

3 Maumenee AE. The influence of donor-recipient sensitization on corneal grafts. Am f Ophthalmol 1951;34:142-52.

4 Niederkorn JY. Animal models of corneal transplantation. In: Zierhut M, Pleyer U, Thiel H-J, eds. Immunology of corneal transplantation. Buren, Netherlands: Aeolus Press, neal transplantat

5 Khodadoust AA. Penetrating keratoplasty in the rabbit. $\mathrm{Am}$ f Ophthalmol 1968;66:899-905.

6 Gronemeyer U. Method of the interlamellar keratoplasty in rats. Graefes Arch Klin Exp Ophthalmol 1972;185:75-82.

7 Williams KA, Coster DJ. Penetrating corneal transplantation in the inbred rat: a new model. Invest Ophthalmol 1985;26:23-30.

8 Streilein JW, McCulley J, Niederkorn JY. Heterotopic corneal grafting in mice: a new approach to the study of corneal alloimmunity. Invest Ophthalmol 1982;23:489-500.

9 Chandler JW, Ray-Keil L, Gillette TE. Experimental corneal allograft rejection: description of a murine model and a new hypothesis of immunopathogenesis. Curr Eye Res 1982;2:387-97.

10 She S-C, Steahly LP, Moticka EJ. A method for performing full-thickness, orthotopic, penetrating keratoplasty in the mouse. Ophthalmic Surg 1990;21:781-5.

11 Sonoda Y, Streilein JW. Orthotopic corneal transplantation in mice-evidence that the immunogenetic rules of rejection do not apply. Transplantation 1992;54:694-704.

12 Joo C-K, Pepose JS, Stuart PM. T-cell mediated responses in a murine model of orthotopic corneal transplantation. Invest Ophthalmol Vis Sci 1995;36:1530-40.

13 Moticka EJ, She S-C. Comparison of failure rates of orthotopic corneal grafts using three different grafting procedures. Curr Eye Res 1989;8:813-20.

14 Gronemeyer U, Pulhom G, Muller-Ruchholtz W. Allogeneic corneal grafting in inbred strains of rats. Histology of graft reaction. Graefes Arch Klin Exp Ophthalmol 1978;208:24762.

15 Tuberville AW, Foster CS, Wood TO. The effect of donor cornea epithelium removal on the incidence of allograft rejection reactions. Ophthalmology 1983;90:1351-6.

16 Stulting RD, Waring GO, Bridges WZ, Cavanagh HD. Effect of donor epithelium on corneal transplant survival. Ophthalmology 1988;95:803-12.

17 Khodadoust AA, Silverstein AM. Transplantation and rejection of individual cell layers of the cornea. Invest $O p h$ thalmol 1969;8:1180-95.

18 Aldredge OC, Krachmer JH. Clinical types of corneal transplant rejection: their manifestation, frequency, preoperative correlates, and treatment. Arch Ophthalmol 1981;99:599604 .

9 Wilson SE, Kaufman HE. Graft failure after penetrating keratoplasty. Surv Ophthalmol 1990;34:325-56.

20 Rinne JR, Stulting RD. Current practices in the prevention and treatment of corneal graft rejection. Cornea 1992;11: $326-8$.

21 Figueiredo FC, Nicholls SM, Easty DL, Shimeld C. Cells infiltrating the corneal epithelium after penetrating keratoplasty in the rat. Invest Ophthalmol Vis Sci 1993;34:1101.

22 Yao YF, Inoue Y, Miyazaki D, Shimomura Y, Ohashi Y, Tano Y. Ocular resurfacing and alloepithelial rejection in a murine keratoepithelioplasty model. Invest Ophthalmol Vis Sci 1995;36:2623-33.

23 Driebe WT, Park JY. Epithelial rejection rings. Arch Ophthalmol 1997;115:938-9.

24 Abbas AK, Lichtman AH, Pober JS. Cellular and molecular immunology. 1st ed. Philadelphia: Saunders, 1991:227. 